Draft version OCtober 9, 2018

Preprint typeset using $\mathrm{IAT}_{\mathrm{E}} \mathrm{X}$ style emulateapj v. 5/2/11

\title{
A FIRST-LOOK ATMOSPHERIC MODELING STUDY OF THE YOUNG DIRECTLY-IMAGED PLANET-MASS COMPANION, ROXS 42Bb
}

\author{
Thayne Currie $^{1}$, Adam Burrows ${ }^{2}$, Sebastian Daemgen ${ }^{1}$ \\ Draft version October 9, 2018
}

\begin{abstract}
We present and analyze $J K_{s} L^{\prime}$ photometry and our previously published $H$-band photometry and $K$-band spectroscopy for ROXs $42 \mathrm{Bb}$, an object Currie et al. (2014) first reported as a young directly imaged planet-mass companion. ROXs 42Bb exhibits IR colors redder than field L dwarfs but consistent with other planet-mass companions. From the $\mathrm{H}_{2} \mathrm{O}-2$ spectral index, we estimate a spectral type of $\mathrm{L} 0 \pm 1$; weak detections/non-detections of the $C O$ bandheads, $\mathrm{NaI}$, and $\mathrm{CaI}$ support evidence for a young, low surface gravity object primarily derived from the $H_{2}(\mathrm{~K})$ index. ROXs $42 \mathrm{Bb}$ 's photometry/K-band spectrum are inconsistent with limiting cases of dust-free atmospheres $(C O N D)$ and marginally inconsistent with the AMES/DUSTY models and the BT-SETTL models. However, ROXS $42 \mathrm{Bb}$ data are simultaneously fit by atmosphere models incorporating several micron-sized dust grains entrained in thick clouds, although further modifications are needed to better reproduce the $K$-band spectral shape. ROXs 42Bb's best-estimated temperature is $T_{\text {eff }} \sim 1950-2000 K$, near the low end of the empirically-derived range in Currie et al. (2014). For an age of $\sim 1-3 \mathrm{Myr}$ and considering the lifetime of the protostar phase, ROXs 42Bb's luminosity of $\log \left(L / L_{\odot}\right) \sim-3.07 \pm 0.07$ implies a mass of $9_{-3}^{+3} M_{J}$, making it one of the lightest planetary mass objects yet imaged.
\end{abstract}

Subject headings: planetary systems, stars: individual: ROXs 42B

\section{INTRODUCTION}

ROXs $42 \mathrm{Bb}$ is a directly-imaged planetary-mass companion to the binary $\mathrm{M}$ star and likely $\rho$ Ophiuchus member, ROXs $42 \mathrm{~B}$, first reported as such by Currie et al. $(2014)^{3}$. The companion at a projected separation of $\approx 157 \mathrm{AU}$, intermediate between the separations of HR 8799 bcde, GJ 504 b, and HD 95086 b (15$70 A U$ Marois et al. 2008, 2010; Kuzuhara et al. 2013; Rameau et al. 2013) and much wider-separation planetmass companions like 1RXJ 1609 B (Lafrenière et al. 2008, 2010). Based on ROXs 42Bb's near-IR brightness and the shape of its $K$-band spectrum, Currie et al. (2014) show that ROXs 42Bb's mass is likely below the deuterium-burning limit $\left(\mathrm{M} \sim 9_{-3}^{+6} M_{J}\right)$.

Constraints on ROXs 42Bb's atmospheric properties are limited thus far. Currie et al. (2014) focused only on simple, empirical comparisons to ROXs 42Bb's nearIR photometry and $K$-band spectrum establishing it as a low surface gravity, late M/early L dwarf planetmass companion, reporting a wide range of possible temperatures $\left(T_{\text {eff }}=1800-2600 \mathrm{~K}\right)$ and surface gravities $(\log (\mathrm{g})=4.0 \pm 0.5)$. Bowler et al. (2014) add $J$ and $H$ band spectra, independently supporting the conclusion from Currie et al. (2014) that ROXs $42 \mathrm{Bb}$ is a low surface gravity/mass companion. But they did not derive a temperature or gravity from template

\footnotetext{
${ }^{1}$ Department of Astronomy and Astrophysics, University of Toronto, 50 St. George St., Toronto, ON, Canada

${ }^{2}$ Department of Astrophysical Sciences, Princeton University

${ }^{3}$ ROXs $42 \mathrm{Bb}$ was first identified as a point source in the Ophiuchus binary survey from Ratzka et al. (2005). Currie et al. (2014) first publicly identify it as a bound, planet-mass companion. Independent work announced, accepted for publication, and published after Currie et al. (2014)'s study identify ROXs 42Bb and come to similar conclusions about its nature (Kraus et al. 2014; Bowler et al. 2014).
}

spectra comparisons nor from atmospheric modeling. The shape of near-IR spectra and near-IR $\left(H / H-K_{s}\right)$ magnitudes/colors are sensitive to temperature and/or surface gravity (Luhman et al. 2004; Allers et al. 2007; Leggett et al. 2010; Stephens et al. 2009; Currie et al. 2013; Canty et al. 2013). However, atmosphere model fits to planet-mass companions focused on the narrow wavelength coverage we presented for ROXs $42 \mathrm{Bb}$ may yield model parameters that are either unphysical or are inconsistent with data obtained at other wavelengths (e.g. Mohanty et al. 2007; Barman et al. 2011).

Data obtained over a wider wavelength baseline, in particular including thermal IR data, can better identify the plausible range of substellar object atmosphere parameters (Stephens et al. 2009) and yield a luminosity estimate. Comparing this luminosity substellar object evolutionary models then yields a mass estimate. A better determined mass for ROXs $42 \mathrm{Bb}$ may also clarify how the companion fits within the context of other imaged planetary-mass companions (Currie et al. 2014).

In this paper, we perform a first-look atmospheric modeling study of ROXs 42Bb. Our analyses combine previously published photometry/spectroscopy with new photometry for ROXs $42 \mathrm{Bb}$ and compare these data to those for other substellar objects. We further analyze the ROXs $42 \mathrm{Bb}$ spectrum to identify major chemical constituents and place additional constraints on the object's spectral type from gravity-independent indices and on its surface gravity from gravity sensitive absorption features. Using planet/brown dwarf atmosphere models defining limiting cases and sophisticated cloud models, we simultaneously fit photometric and spectroscopic data to better clarify ROXs 42Bb's temperature, surface gravity, luminosity and mass.

\section{DATA}




\subsection{New Data}

\subsubsection{Observations and Image Processing}

We reduced $J, K_{s}$ and $L^{\prime}$ data for ROXs 42B obtained from the Keck Observatory Archive (KOA), taken on 22 June 2011 with the NIRC2 'narrow' camera (9.952 mas pixel $^{-1}$ Yelda et al. 2010). All new data were taken with good adaptive optics corrections in classical imaging, and in dither/nod patterns to remove the sky background. The field of view was $\approx 10$ arc-seconds on a side. Basic image processing steps were identical to those carried out for previously published ROXs $42 \mathrm{Bb}$ photometry (Currie et al. 2014). Briefly, we first sky-subtracted each image from a median combination of images obtained at other dither positions. Then, we identified and removed hot/cold/bad pixels, corrected each image for distortion using the nirc2dewarp.pro IDL routine, copied each to larger blank image, and registered the images to a common center (Currie et al. 2012a,b). We then subtracted off a $2 \mathrm{D}$ radial profile of the primary to remove the halo light from each image. After these steps, we median-combined together our set of images and rotated the combined image to the north-up position $\left(P A_{\text {north }}\right.$ $=-0.252^{\circ}$; Yelda et al. 2010).

Figure 1 displays the combined $J$ (left), $K_{s}$ (middle), and $L^{\prime}$ images, revealing ROXs $42 \mathrm{Bb}$ at an angular separation of $\approx 1^{\prime \prime}$. 17 and position angle of $\approx 270^{\circ}$. Additionally, each data set reveals the second candidate companion, "ROXs $42 \mathrm{~B}$ c", at roughly 0 ".5 separation, although the $L^{\prime}$ detection is noticeably weaker than detections at $J$ and $K_{s}$. ROXs $42 \mathrm{~B}$ is defined as a close binary $\left(\Delta \theta \approx 00^{\prime \prime} 05\right)$ identified from lunar occultation data (e.g. Simon et al. 1995); however, we fail to resolve the two components (see also Currie et al. 2014; Kraus et al. 2014).

\subsubsection{Photometry}

We largely follow previous methods used to extract photometry for the ROXs $42 \mathrm{~B}$ system as described in Currie et al. (2014). For each data set, we measure the integrated signal for both the primary (in a mediancombined registered image) and the companions (in a median-combined radial-profile subtracted image) in an aperture sized to the full-width half-maximum of the primary star and define and subtract off the background signal in a surrounding annulus. As a separate check, we extracted photometry assuming a zero background signal and with slightly different sized apertures. Our photometric uncertainties incorporate the intrinsic SNR of the companions, the SNR of the primary star, and differences in brightness measurements adopting different aperture sizes and background treatments.

These procedures yield a companion-to-primary contrast of $\Delta \mathrm{J}=7.00 \pm 0.11, \Delta K_{s}=6.33 \pm 0.06$, and $\Delta L^{\prime}=5.64 \pm 0.06$ for ROXs $42 \mathrm{Bb}$. For the putative "ROXs 42Bc" object, we derive $\Delta \mathrm{J}=6.69 \pm 0.12, \Delta K_{s}$ $=6.78 \pm 0.07$, and $\Delta L^{\prime}=6.76 \pm 0.20$. Our K-band photometry for ROXs $42 \mathrm{Bb}$ agrees with that derived from older Keck/NIRC2 data reported in Currie et al. (2014). Kraus et al. (2014)'s $J$ and $L^{\prime}$ photometry agrees with our values for ROXs $42 \mathrm{Bb}$. One of their $K_{s}$ band estimates agree with ours while the other two measurements of ROXs $42 \mathrm{Bb}$ are significantly too faint or bright and in fact inconsistent with one another given their reported photometric errors and adopted limits on stellar variability, discrepancies potentially due to their aperture photometry methods (see Section 2.2).

To flux-calibrate the companions' photometry in $J$ and $K_{s}$, we simply adopt the 2MASS measurements as reported before: $9.906 \pm 0.02$ and $8.671 \pm 0.02$. To estimate the $L^{\prime}$ brightness of ROXs $42 \mathrm{~B}$, we adopt the intrinsic colors for young pre-main sequence stars listed in Pecaut et al. (2013), assume that the $K_{s}-L^{\prime}$ color is between the predicted $K_{s^{-}} W 1$ and $K_{s^{-}} W 2$ 2MASS/WISE colors $\left(\lambda_{o}(W 1, W 2)=3.4,4.6 \mu \mathrm{m}\right)$ or $K_{s}-L^{\prime}=0.14$, and assume a $0.05 \mathrm{mag}$ uncertainty in $K_{s^{-}} L^{\prime}$ color from the difference between the two 2MASS/WISE color:4 Adopting the Cardelli et al. (1989) reddening relations and assuming $A_{V}=1.9$ (Currie et al. 2014) then yields a predicted $L^{\prime}$ magnitude of $8.42 \pm 0.05$. This value also agrees with a straight or weighted average of ROXs 42B's measured $\mathrm{K}_{s}$-W1 and $\mathrm{K}_{s}$-W2 colors (8.40-8.43 \pm $0.03)$. To then estimate the dereddened absolute magnitudes for ROXs $42 \mathrm{Bb}$ we assume a distance of $135 \pm 8 p c$ (Mamajek 2008) and the same values for the extinction and reddening law.

\subsection{Previously Published Data}

To these data we add VLT/SINFONI spectra and $H$ band photometry previously published in Currie et al. (2014). We measure the primary-to-companion contrast for ROXs $42 \mathrm{Bb}$ as $\Delta H=6.86 \pm 0.05$. Given the 2MASS $H$-band photometric measurement of $\mathrm{m}_{H}=9.017 \pm$ 0.020 , we derive an apparent magnitude of $15.88 \pm 0.06$ and a dereddened absolute magnitude of $9.87 \pm 0.14$.

As noted in Currie et al. (2014) the putative "ROXs $42 \mathrm{Bc}$ " is of comparable brightness in $H$ band. Quantitatively, we derive a contrast of $\Delta H=6.94 \pm 0.09$ and an apparent magnitude of $15.96 \pm 0.09$. These values significantly disagree with the contrast estimate by Kraus et al. (2014) who claim $\Delta \mathrm{H}=6.20$. With the background signal largely removed from a radial profile subtraction or explicitly measured in a median-combined frame, "ROXs $42 \mathrm{Bc} "$ clearly cannot be $\sim$ twice as bright as ROXs $42 \mathrm{Bb}$

Table 1 summarizes our photometric measurements for both ROXs $42 \mathrm{Bb}$ and "ROXs $42 \mathrm{Bc}$ ". While both companions have similar $H$-band contrasts and apparent magnitudes, their observed colors are quite different. In particular, ROXs $42 \mathrm{Bb}$ has a $J-L^{\prime}$ color of 2.86 , significantly redder than that of "ROXs 42Bc" (1.42), a difference largely owing to the latter's significantly fainter $L^{\prime}$ flux density.

Additional arguments show that "ROXs $42 \mathrm{Bc}$ " is likely a reddened background object. Besides being significantly fainter at $L^{\prime}$, it is about 0.45 mags fainter at $K_{s}$ band and $\sim 0.31$ mags brighter at $J$. If the line-of-sight extinction between us and "ROXs $42 \mathrm{Bc}$ " is in the $A_{V}=$

4 While ROXs $42 \mathrm{~B}$ is a binary with unequal mass components, the brightness ratio at $K_{s}$ band is $\sim 2$ to 1 , and the $K_{s}-\mathrm{W} 1$ and $K_{s}$-W2 colors for young M0-M5 dwarfs varies by less than 0.1 and 0.25 magnitudes. So the $K_{s}-L^{\prime}$ color should reflect that of the brighter component. There is no evidence of ROXs42B being variable in brightness.

5 The implied relative brightness of "ROXs $42 \mathrm{Bc}$ " is higher in raw counts from median-combined, registered images without proper background subtraction, with peak values of roughly $\sim 1225$ cts. vs. 810 cts for ROXs $42 \mathrm{Bb}$. However, the background signal around "ROXs $42 \mathrm{Bc}$ " is 500 cts higher. 
4-6 range consistent with it being behind the filamentary structure containing ROXs $42 \mathrm{~B}$, then the object dereddens to the colors expected for an early-type star. For example, assuming $A_{V}=4.5$ yields $\mathrm{m}(J)=15.33 \pm 0.12$, $\mathrm{m}(H)=15.11 \pm 0.09, \mathrm{~m}\left(K_{s}\right)=14.94 \pm 0.07$ and $\mathrm{m}\left(L^{\prime}\right)$ $=14.93 \pm 0.20$, consistent with the colors for an A0 to mid F star (see Pecaut et al. 2013; Currie et al. 2010). Thus, as previously suggested in Currie et al. (2014) and argued independently by Kraus et al. (2014), our analysis shows that "ROXs $42 \mathrm{Bc}$ " is likely not a second planetary-mass companion orbiting ROXs 42B.

\section{DIRECT CONSTRAINTS ON ROXS 42Bb'S ATMOSPHERE}

\subsection{IR Colors}

To compare the near-to-mid IR photometric properties of ROXs $42 \mathrm{Bb}$ with those for other substellar objects, we follow similar analysis in Currie et al. (2013), using the Leggett et al. (2010) sample of field brown dwarfs with spectral classes between M7 and T5. To these objects, we add the directly-imaged planetary-mass companions/candidates listed in their Table 4 using photometric measurements listed in their Table 5. For clarity, we do not include young objects with inferred masses above the deuterium-burning limit: these tend to be much more luminous in each infrared filter than ROXs 42Bb.

Figure 2 compares the $J / J-K_{s}$ and $H / H-L^{\prime}$ colormagnitude position of ROXs $42 \mathrm{Bb}$ (blue diamond) to those positions for older, field brown dwarfs (black/grey dots) and other planetary-mass companions (aqua squares). Currie et al. (2014) found that the near-IR $H / H-K_{s}$ color-magnitude diagram position for ROXs $42 \mathrm{Bb}$, like that for directly-imaged planets $\beta$ Pic b and HR 8799 bcde (Marois et al. 2008; Currie et al. 2011, 2013), was discrepant compared to the field MLT dwarf sequence. The same trends persist when we consider $J$ and $L^{\prime}$ data, as ROXs $42 \mathrm{Bb}$ is $\sim 0.5$ mags redder than the field sequence in both $J-K_{s}$ and $H / H-L^{\prime}$.

Compared to the planet-mass companions plotted, ROXs 42Bb's $J / J-K_{s}, H / H-L^{\prime}$, and $H / H-K_{s}$ (Currie et al. 2014) color-magnitude diagram position bears the strongest resemblance to $6-16 M_{J} \mathrm{M} / \mathrm{L}$ transition objects GSC 06214B and USco CTIO 108 B (Ireland et al. 2011; Bejar et al. 2008). These objects have masses of $\sim 6-16 M_{J}$, and spectral types of M9.5L0. Given their slightly older but qualitatively similar ages of $\approx 5-10$ Myr (Pecaut et al. 2012; Bowler et al. 2011), we expect they should serve as good indicators for ROXs 42Bb's spectral type and inferred mass.

\subsection{Spectral Analysis}

In Currie et al. (2014), we focused on simple, empirical comparisons to the ROXs $42 \mathrm{Bb}$ spectral shape to estimate its spectral type and assess evidence for low surface gravity (see also Bowler et al. 2014). Here, we further analyze the spectrum (Figure 3) to estimate its spectral type from gravity independent (or weakly dependent) indices (Allers and Liu 2013; Bonnefoy et al. 2013), identify chemical species present, and corroborate evidence for low surface gravity from alternate diagnostics (Canty et al. 2013).

\subsubsection{Spectral Type}

To estimate the spectral type of ROXs $42 \mathrm{Bb}$, we compute the $\mathrm{H}_{2} 0-2$ index, defined by Slesnick et al. (2004) as the $2.04 \mu \mathrm{m}$ to $2.15 \mu \mathrm{m}$ flux ratio. This index appears well correlated with spectral type over the range enclosing ROXs 42Bb's likely value and for substellar objects in the Orion Nebula Cluster, which are roughly coeval with ROXs $42 \mathrm{Bb}$. As this is an estimate of the strength of the broad $\mathrm{H}_{2} \mathrm{O}$ absorption feature at $\approx 2 \mu \mathrm{m}$, not a narrow line feature, we compute the index for a smoothed, binned version of the spectrum that we later use for atmospheric modeling $(R \sim 650$, see Sect. 4$) 6$.

We measure ROXs $42 \mathrm{Bb}$ 's $\mathrm{H}_{2} \mathrm{O}-2$ index to be $\left[\mathrm{H}_{2} \mathrm{O}\right]$ $\sim 0.87$. To estimate a spectral type from this index, we consider identical measurements for young substellar objects in Upper Scorpius (Lodieu et al. 2008), the relationship mapping between these two quantities in Bonnefov et al. (2013), and the polynomial fit from Allers and Liu (2013). We then take the average of these values as our spectral type and the dispersion in these values as our uncertainty.

The Allers and Liu (2013) polynomial fit yields a spectral type of L0, whereas the Bonnefor et al. (2013) relationship yields M9 and Lodieu et al. (2008) empirical scale yields L0-L1. From these comparisons, we then estimate ROXs $42 \mathrm{Bb}$ 's spectral type to be $\mathrm{L} 0 \pm 1$ subclass. Thus, our estimate for ROXs 42Bb's spectral type is consistent with simple empirically-based estimates from Currie et al. (2014) and Bowler et al. (2014) of M8-L0 and $\mathrm{L} 1 \pm 1$, respectively.

\subsubsection{Chemical Composition and Gravity-Sensitive Features}

The spectra of late M/early $\mathrm{L}$ dwarfs in $K$-band are characterized by strong $\mathrm{H}_{2} \mathrm{O}$ absorption from the blue end of the bandpass through $\approx 2.1 \mu \mathrm{m}$, collisionallyinduced absorption of $\mathrm{H}_{2}\left(\mathrm{CIA} \mathrm{H}_{2}\right)$ at $2.18-2.28 \mu \mathrm{m}$ (Kirkpatrick et al. 2006; Saumon et al. 2012), prominent $\mathrm{CO}$ lines starting at $2.29 \mu \mathrm{m}$, and $\mathrm{H}_{2} \mathrm{O}$ absorption longwards of $2.3 \mu \mathrm{m}$. Field objects near the M/L transition have weaker $\mathrm{Na}$ I doublet lines at 2.206 and $2.209 \mu \mathrm{m}$ and Ca I triplet emission $(2.261,2.263$, and $2.265 \mu \mathrm{m}$ ) than earlier spectral types (earlier than M7; Cushing et al. 2005). These features may weaken further for younger, lower surface gravity counterparts (Canty et al. 2013).

In the ROXs $42 \mathrm{Bb}$ spectrum (Figure 3), we see clear evidence for $\mathrm{H}_{2} \mathrm{O}$ absorption at the blue and red ends, similar to that seen in both the $H$ and $K$-band spectrum presented in Bowler et al. (2014). Our spectrum also exhibits weak CIA $H_{2}$ responsible for the rather red 2.17-2.24 $\mu \mathrm{m}$ slope indicative of very low surface gravity planet-mass companions (c.f. Kirkpatrick et al. 2006; Canty et al. 2013; Currie et al. 2014). Likewise, ROXs $42 \mathrm{Bb}$ exhibits CO bandhead structure at $\lambda>2.29 \mu \mathrm{m}$. The spectrum is particularly noisy near the bandpass limits and thus we do not look for evidence of $\mathrm{Ca} \mathrm{I}$ absorption at $\sim 1.98 \mu \mathrm{m}$.

To augment our analysis of CIA $\mathrm{H}_{2}$ in Currie et al. (2014), we look for evidence of other, more weakly gravity sensitive features $\mathrm{Na} \mathrm{I} / 2.21 \mu \mathrm{m}$ and $\mathrm{Ca} \mathrm{I} / 2.26 \mu \mathrm{m}$ studied in Canty et al. (2013). While the CO bandhead is clearly detected, the features are particularly weak. Using the IRAF task splot, we calculate an equivalent width of $W_{\mathrm{CO}(2-0)} \sim 8 \pm 2 \AA$ at $2.29 \mu \mathrm{m}$, comparable to

\footnotetext{
${ }^{6}$ We derive similar estimates for the raw spectrum.
} 
or smaller than any young object studied in Canty et al. (2013) and much smaller than equilvalent widths for field late $\mathrm{M}$ /early $\mathrm{L}$ dwarfs. We do see local minima at the positions of the $\mathrm{Na} \mathrm{I}$ and $\mathrm{Ca}$ I features with equivalent widths of $\approx 2 \AA$ and $1 \AA$. However, they are difficult to distinguish from the noise in the spectrum and thus their detections are marginal at best. These estimates consistent with results for $\mathrm{ONC} \mathrm{M} / \mathrm{L}$ transition objects but inconsistent/marginally consistent with measurements for field objects (Canty et al. 2013). Thus, the line strengths of these secondary gravity diagnostics agree with our general conclusion based on the $\mathrm{H}_{2}(\mathrm{~K})$ index: ROXs $42 \mathrm{Bb}$ is a low surface gravity substellar object.

\section{ATMOSPHERIC MODELING}

Atmosphere Models Considered - To explore ROXs 42Bb's atmospheric properties, we compare the object's broadband photometry and $K$-band spectrum to predictions from planet atmosphere models adopting a range of effective temperatures, surface gravities, and cloud/dust prescriptions. Table 2 summarizes our parameter space. We consider two limiting cases for dust/clouds in model atmospheres, the cloud/dust free COND models (Baraffe et al. 2003) and the AMES/DUSTY models which assume that submicron-sized dust grains are suspended everywhere in the atmosphere. Then we compare the ROXs $42 \mathrm{Bb}$ data to the BT-Settl model atmospheres (Allard et al. 2012) and our own (Currie et al. 2013), both of which adopt cloud models. The COND and DUSTY models cover a wide range in temperature and gravity $\left(T_{\text {eff }}=1600\right.$ $2800 \mathrm{~K}$ where $\Delta T_{\text {eff }}=100 \mathrm{~K}, \log (\mathrm{g})=3.5-5$ where $\Delta(\log (\mathrm{g}))=0.5)$. The BT-Settl model grid is smaller, as is our own model grid (hereafter referred to as "the Burrows grid").

Fitting Method - To model photometry, we simply compare the measured flux densities with predicted ones from the model spectra convolved with each filter function. The COND, DUSTY, and BT-Settl grid resolutions are at significantly higher resolution than the data $(R \approx$ $4000)$, whereas the Burrows grid is at lower resolution $(R$ $\sim 650$ ). To provide more direct quantitative comparisons amongst the model fits, we smooth and rebin the other atmosphere models to the Burrows model grid. Similarly, we rebin the extracted ROXs $42 \mathrm{Bb}$ spectrum to the Burrows grid resolution]. Our modeled wavelength range is 1.95 to $2.45 \mu \mathrm{m}$.

To conservatively estimate errors in each spectral channel, we model and subtract off the pseudo-continuum, compute the rms noise in a moving box centered on each channel. Furthermore, we consider systematic errors in the spectral extraction, defined as differences in the spectrum's flux density as a result of various measurements of the spatially variable background. Finally, we consider errors in the spectra measurements from uncertainties in dereddening the spectrum that are ultimately due to uncertainties in the spectral type of ROXs 42B (K5-M1; see footnote in Currie et al. 2014). The signal-to-noise ratio of the binned spectrum varies from a peak of $\approx 25$ to $\sim 5$ for the bluest and reddest channels. Thus, our

7 We found nearly identical modeling results for the COND, DUSTY, and BT-Settl grid if we simply degraded their resolution to that of the data. model fitting is by far most sensitive to matches near the peak of the spectrum $(2.1-2.2 \mu \mathrm{m})$ than the edges, which could be affected by telluric contamination.

We treat the planet radius as a free parameter, varying it between $0.9 R_{J}$ and $3 R_{J}$. We identify the set of models formally consistent with the spectra or photometry at the $95 \%$ confidence limits given the number of degrees of freedom (Currie et al. 2011). Although we adopt the dereddened absolute magnitude to compare photometry with each model, we adopt only the measured photometric error (listed in fourth column of Table 1) for model fitting and incorporate our distance uncertainty in the uncertainty estimate for the planet radius. We derive results for photometry and $K$-band spectroscopy separately and then compare them to identify the subset of models consistent with both.

\subsection{Photometry Fitting Results}

Table 3 summarizes our model fitting results. Figure 4 displays the $\chi^{2}$ distributions for our photometric model fits while Figure 5 shows the best-fit model spectrum and predicted photometry (magenta lines) with the photometric data overplotted. The dust-free $C O N D$ models fail to reproduce the photometry (Figure 4 and 5 , topleft panels $)$ at a statistically significant level $\left(\chi_{\min }^{2} \sim\right.$ $35.9 \gg>\chi_{95 \%}^{2} \sim 9.5$ ). The BT-Settl models (same figures, bottom-left panels) perform better but include only one model $\left(T_{\text {eff }}=1800, \log (\mathrm{g})=3.5\right)$ consistent with the data at the $95 \%$ confidence limit.

Models incorporating substantial atmospheric dust and/or thick clouds best reproduce ROXs 42Bb's photometry. The DUSTY models quantitatively yield the better fits than either COND or BT-Settl (top-right panel $)$, with a $\chi^{2}$ minima of $\sim 2.47\left(T_{\text {eff }}=1900 \mathrm{~K}\right.$, $\log (\mathrm{g})=4$ ) and models spanning $T_{\text {eff }} \sim 1800-1900 \mathrm{~K}$ consistent within the $95 \%$ confidence limit. The Burrows models reproduce the data even better (bottom-right panel) for $T_{\text {eff }}=1800-2000 K$ and $\log (\mathrm{g})=3.6-4$ and $T_{\text {eff }}=1900-2000 \mathrm{~K}$ with $\log (\mathrm{g})=3.4\left(\chi_{\min }^{2}=0.57\right)$. While empirical comparisons to ROXs $42 \mathrm{Bb}$ 's spectrum allowed temperatures of 1800-2600 K, modeling the object's photometry strongly favors the lower part of this range.

\subsection{Spectroscopy Fitting Results}

Figures 6 and 7 display the $\chi^{2}$ distributions for our spectroscopic model fits and best-fit model with the $K$ band spectrum overplotted. The COND and DUSTY model spectra yield significant fits only at high temperatures $\left(T_{\text {eff }}=2600-2800 \mathrm{~K}\right)$. The BT-Settl models fit the spectrum well at $1700-1900 \mathrm{~K} / \log (\mathrm{g})=4-5$ and $2400 K / \log (\mathrm{g})=3.5$, accurately reproducing the shape of most of the $K$-band peak and its location. While the fit from the Burrows models tends to predict too flat of a $K$-band spectrum compared to BT-Settl, models with $T_{\text {eff }}=1950-2100 \mathrm{~K}$ and $\log (\mathrm{g})=3.4-3.8$ are consistent with the spectrum to within the $95 \%$ confidence limit8.

\subsection{Derived Best-Fit Atmosphere Modeling Parameters}

${ }^{8}$ While there is a slight preference for lower surface gravity models, the $\chi^{2}$ value much more strongly depends on temperature. 
Figures 8 and 9 and the righthand columns of Table 3 clarify which model parameter space matches the ROXs $42 \mathrm{Bb}$ photometry and $K$-band spectrum simultaneously. Although some COND and DUSTY models fit the $K$-band spectrum, the same models fail to reproduce the ROXs $42 \mathrm{Bb}$ photometry. Similarly, the $C O N D$ and $D U S T Y$ models best fitting the photometry fail to reproduce the $K$-band spectral shape. Thus, neither of the model limiting cases can simultaneously fit photometry and spectra.

The inferred temperatures for BT-Settl models that best fit the spectrum and the photometry are similar. However, as with the COND and DUSTY models, the specific BT-Settl models yielding fits to within the $95 \%$ confidence limit for the spectra fail to match the photometry (Figure 8) and vice-versa (Figure 9), owing to the model predicting too narrow of a $2.2 \mu \mathrm{m}$ peak. In contrast to all other models, the Burrows models are able to yield some parameter space that fits both the ROXs $42 \mathrm{Bb}$ photometry and spectroscopy at the $95 \%$ confidence limit. For instance, the $T_{\text {eff }}=2000 \mathrm{~K}, \log (\mathrm{g})$ $=3.6$ model that best fits the spectrum also agrees with the photometric data to within the $95 \%$ confidence limit (Figure 8, bottom-right panel).

To derive best-estimated atmospheric parameters, we focus on the Burrows thick cloud models that simultaneously fit the photometry and $K$-band spectrum: $T_{\text {eff }}=$ $1950-2000 K, \log (\mathrm{g})=3.4-3.8$. Thus, spectral analysis (Sect 3.2) and atmospheric modeling (Sect. 4.1-4.2) combined shows ROXs $42 \mathrm{Bb}$ to have a very dusty/cloudy, low surface gravity L0 dwarf atmosphere with $T_{\text {eff }}=$ 1950-2000 K.

\subsection{Inferred Properties from Best-Fitting Models}

The models matching ROXs 42Bb's photometry and spectroscopy allow us to derive an estimated radius for the object. Compared to the nominal radii for a given $T_{\text {eff }}$ and $\log (\mathrm{g})$ assumed from the Burrows et al. (1997) evolutionary models, our fits require radii larger by factors of $1.25-1.53$ for models with $T_{\text {eff }}=1950 \mathrm{~K}$ and $\log (\mathrm{g})=3.4-3.8$. For the $T_{\text {eff }}=2000 \mathrm{~K}$ and $\log (\mathrm{g})=$ 3.4-3.8 models, the scaling factors are smaller: $1.2-1.45$. In each case, the scaling factors that best fit the photometry match those that best fit the spectrum to within $2.5 \%$. We obtain best-fit radii of $R / R_{J} \sim 2.55 \pm 0.20$ for the $T_{\text {eff }}=1950 \mathrm{~K}$ models and $R / R_{J} \sim 2.43 \pm 0.18$ for the $T_{\text {eff }}=2000 \mathrm{~K}$ models, where the errors include consider the dispersion in radius scaling estimates amongst good-fitting models and the distance uncertainty of $\sim$ $5 \%$.

ROXs 42Bb's luminosity-estimated mass, derived from the temperature and radius of our fits, is consistent with the sub-deuterium burning, $9_{-3}^{+6} M_{J}$ estimate from Currie et al. (2014). Luminosity estimates for the $T_{\text {eff }}$ $=1950 \mathrm{~K}$ and $2000 \mathrm{~K}$ models yield identical values: $\log \left(\mathrm{L} / \mathrm{L}_{\odot}\right)=-3.07 \pm 0.07$. Given an estimate age of 2.5-3 Myr for ROXs 42B (Currie et al. 2014), both the Baraffe et al. (2003) and Burrows et al. (1997) "hot start" evolutionary models nominally predict a mass of $10_{-1}^{+2} M_{J}$. Displacing the evolutionary tracks by a characteristic protostar lifetime of $\tau_{\text {Class } 0 / I} \sim 0.5 \mathrm{Myr}$ (Evans et al. 2009) lowers the masses to $9_{-1}^{+1.5} M_{J}$. If instead ROXs 42B is 1 Myr old like much of the $\rho$ Oph complex, ROXs 42Bb's mass is $\approx 6 M_{J}$.

Considering these uncertainties, we estimate a mass of $9_{-3}^{+3} M_{J}$ for ROXs $42 \mathrm{Bb}$. We derive consistent (slightly lower, much higher) mass estimates if we take the surface gravity and radii of our best-fit models at face value: for $\log (\mathrm{g})=3.6(3.4,3.8)$ we derive $M \approx 9.1-10.4 M_{J}(6-7.3$ $\left.M_{J}, 14.3-15.8 M_{J}\right)$.

\section{DISCUSSION}

Our analysis of ROXs 42Bb's 1-4 $\mu m$ photometry and $K$-band spectrum provides strong evidence that its atmosphere is dusty, with an effective temperature of $\sim$ $2000 K$. ROXs 42Bb's luminosity of $\log \left(\mathrm{L} / \mathrm{L}_{\odot}\right) \sim-3.07$ \pm 0.07 , when compared to evolutionary models, implies a mass of 6-12 $M_{J}$. Thus, our atmosphere modeling supports earlier, largely empirically-driven arguments from Currie et al. (2014) that ROXs $42 \mathrm{Bb}$ is a sub-deuterium burning, planet-mass object.

Despite our success in identifying some acceptably fitting models as determined by a $\chi^{2}$ statistic, ROXs $42 \mathrm{Bb}$ 's atmosphere still presents significant modeling challenges. In particular, no model yet reproduces both the photometry and the triangular-shaped pseudo-continuum at $K$ band peaked at $2.24 \mu \mathrm{m}$. The primary mismatches between the best-fit Burrows models and the spectrum are that the models predict too flat of a spectrum, resulting in an overpredicted flux density at $2-2.1 \mu \mathrm{m}$ and underpredicted flux density over the $2.25-2.3 \mu \mathrm{m}$ region. As $\mathrm{H}_{2} \mathrm{O}$ absorption controls the shape of the spectrum at 2 $2.15 \mu \mathrm{m}$ and CIA $\mathrm{H}_{2}$ controls the slope of the spectrum at $\sim 2.2-2.3 \mu \mathrm{m}$, different treatments of these opacity sources may improve fits to the datd?.

Our explored model parameter space is limited in important ways that may slightly modify the best inferred atmospheric properties. For instance, as our model fits are only weakly sensitive to gravity, we adopted a narrow range of $\log (\mathrm{g})=3.4-4$. Best-fit atmosphere models typically required larger radii by $20-30 \%$ compared to their nominal values, which are tied to the Burrows et al. (1997) planet/brown dwarf luminosity evolution models, where the rescaling was smaller for lower gravity. Exploring even lower gravities (e.g. $\log (\mathrm{g})=3-3.2$ ) may yield as good or better fits to the data without rescaling any radii and thus may be preferred. Models with non-solar carbon abundances may provide marginally better fits to the photometry and/or spectra of hot, young planetmass objects (Barman et al. 2011; Galicher et al. 2011; Currie et al. 2013). These issues should be considered to better constrain ROXs 42Bb's atmospheric properties, in particular its gravity and chemistry.

Finally, incorporating new near-IR spectra may further clarify ROXs 42Bb's temperature, gravity, and chemistry. The $\mathrm{H}_{2} \mathrm{O}$ index at the blue edge of $\mathrm{H}$ band (Allers and Liu 2013) provides a gravity insensitive independent check on our $K$-band derived spectral type as does the $\mathrm{H}_{2} \mathrm{O}-1$ index near $J$ band (Slesnick et al. 2004). The depth of $K I$ lines in $J$ band spectra of substellar objects is gravity sensitive (Allers et al. 2007;

\footnotetext{
${ }^{9}$ Note that the Burrows models are not alone in being strained to reproduce some gravity sensitive features in the spectra of young substellar/planet-mass objects. For instance, the $\mathrm{H}_{2}(\mathrm{~K})$ index appears to be a much stronger function of gravity than predicted from the Saumon and Marley models (see Canty et al. 2013).
} 
TABLE 1

NeAR-TO-Mid Infrared Photometry

\begin{tabular}{lllll}
\hline \hline Object & Filter & $\Delta(\mathrm{mag})$ & Apparent Magnitude & Dereddened Absolute Magnitude \\
\hline ROXs 42Bb & $J$ & $7.00 \pm 0.11$ & $16.91 \pm 0.11$ & $10.72 \pm 0.17$ \\
& $H$ & $6.86 \pm 0.05$ & $15.88 \pm 0.05$ & $9.87 \pm 0.14$ \\
& $K_{s}$ & $6.34 \pm 0.06$ & $15.01 \pm 0.06$ & $9.13 \pm 0.14$ \\
"ROXs 42Bc" & $L^{\prime}$ & $5.64 \pm 0.06$ & $14.06 \pm 0.06$ & $8.30 \pm 0.15$ \\
& $J$ & $6.69 \pm 0.12$ & $16.60 \pm 0.12$ & - \\
& $H$ & $6.94 \pm 0.09$ & $15.96 \pm 0.09$ & - \\
& $K_{s}$ & $6.78 \pm 0.07$ & $15.45 \pm 0.07$ & - \\
& $L^{\prime}$ & $6.76 \pm 0.20$ & $15.18 \pm 0.20$ & -
\end{tabular}

Note. - New data is from Keck PID HD242N2 (P.I. A. Kraus). Assuming a reddening of $A_{V}=1.9$ and an intrinsic color of $K_{S}-L^{\prime}=0.14 \pm 0.05$ for ROXs $42 \mathrm{~B}$, we estimate an apparent $L^{\prime}$ magnitude of $8.42 \pm 0.05$. The ROXs $42 \mathrm{Bb} H$ band photometry was previously reported in Currie et al. [2014).

TABLE 2

Atmosphere Modeling Grid

\begin{tabular}{lclcc}
\hline \hline Model & & \multicolumn{4}{c}{ Range } \\
& $T_{\text {eff }}(K)$ & $\Delta T_{\text {eff }}$ & $\log (\mathrm{g})$ & $\Delta(\log (\mathrm{g}))$ \\
\hline Limiting Cases & & & & \\
AMES-COND & $1600-2800$ & 100 & $3.5-5$ & 0.5 \\
AMES-DUSTY & $1600-2800$ & 100 & $3.5-5$ & 0.5 \\
Cloud Models & & & & \\
BT-Settl & $1500-2400$ & 100 & $3.5-5$ & 0.5 \\
Burrows/A4 & $1500-1800,2100-2400$ & 100 & $3.6-4$ & 0.2 \\
& $1900-2000$ & 50 & $3.4-4$ & 0.2 \\
& & & &
\end{tabular}

Luhman et al. 2007) and provides another way to compare ROXs 42Bb's atmosphere with those for other young substellar objects, especially those in Taurus and Upper Scorpius.

We thank Thorsten Ratzka, Ernst De Mooj, and Scott Kenyon for helpful draft comments and the anonymous referee for suggestions improving the presentation and data analyses in our paper. This research has made use of the Keck Observatory Archive (KOA), which is operated by the W. M. Keck Observatory and the NASA Exoplanet Science Institute (NExScI), under contract with the National Aeronautics and Space Administration. TC and SD are supported by McLean Postdoctoral Fellowships.

\section{REFERENCES}

Allard, F., et al., 2001, ApJ, 556, 357

Allard, F., et al., 2012, in EAS Publications Series, Vol. 57. Ed. C. Reyle, C. Charbonnel, \& M. Schultheis, 3-43

Allers, K., et al., 2007, ApJ, 657, 511

Allers, K., Liu, M., 2013, ApJ, 772, 79

Baraffe, I., et al., 1998, A\&A, 337, 403

Baraffe, I., et al., 2003, A\&A, 402, 701

Barman, T., et al., 2011, ApJ, 735, L39

Bejar, V., et al., 2008, ApJ, 673, L185

Bonnefoy, M., et al., 2013, A\&A in press

Bowler, B., et al., 2011, ApJ, 743, 148

Bowler, B., et al., 2014, ApJ in press

Burrows, A., et al., 1997, ApJ, 491, 856

Burrows, A., et al., 2006, ApJ, 640, 1063

Canty, J., et al., 2013, MNRAS, 435, 2650

Cardelli, J., et al., 1989, ApJ, 345, 245

Chauvin, G., et al., 2004, A\&A, 425, 29L

Currie, T., Hernandez, J., et al., 2010, ApJS, 186, 191

Currie, T., Burrows, A., et al., 2011, ApJ, 729, 128

Currie, T., et al., 2012a, ApJ, 760, L32

Currie, T., et al., 2012b, ApJ, 755, L34

Currie, T., et al., 2013, ApJ, 776, 15

Currie, T., et al., 2014, ApJ, 780, L30

Cushing, M., et al., 2005, ApJ, 623, 1115

Evans, N., et al., 2009, ApJS, 181, 321

Galicher, R., et al., 2011, ApJ, 739, L41

10 While a detailed comparison with Bowler et al. (2014)'s $J$ and $H$ spectra is beyond the scope of this paper, they did provide
Ireland, M., et al., 2011, ApJ, 726, 113

Kirkpatrick, D., et al., 2006, ApJ, 639, 1120

Kraus, A., et al., 2014, ApJ in press

Kuzuhara, M., et al., 2013, ApJ, 774, 11

Lafrenière, D., et al., 2008, ApJ, 689, 153L

Lafrenière, D., et al., 2010, ApJ, 719, 497

Leggett, S., et al., 2010, ApJ, 710, 1627

Lodieu, N., et al., 2008, MNRAS, 383, 1385

Luhman, K., Peterson, D., Megeath, S. T., 2004, ApJ, 617, 565

Luhman, K., et al., 2007, ApJ, 659, 1629

Mamajek, E., 2008, AN, 329, 10

Marois, C., et al., 2006, ApJ, 641, 556

Marois, C., et al., 2008, Science, 322, 1348

Marois, C., et al., 2010, Nature, 468, 1080

Mohanty, S., et al., 2007, 657, 1064

Pecaut, M. J., et al., 2012, ApJ, 746, 154

Pecaut, M. J., \& Mamajek, E. E. 2013, ApJS, 208, 9

Ratzka, T., Kohler, R., Leinert, C., 2005, A\&A, 437, 611

Rameau, J., et al., 2013, ApJ, 779, L26

Saumon, D., et al., 2012, ApJ, 750, 74

Simon, M., et al., 1995, ApJ, 443, 625

Slesnick, C., et al., 2004, AJ, 610, 1045

Stephens, D., et al., 2009, ApJ, 702, 154

Yelda, S., et al., 2010, ApJ, 725, 331

some evidence that ROXs 42Bb's spectrum at $J$ and $H$ band bears strong resemblance to young planet-mass objects. Our best-fit at- 
TABLE 3

Model Fitting Results

\begin{tabular}{|c|c|c|c|}
\hline Model & $\begin{array}{l}\text { Photometry } \\
\mathrm{T}_{\text {eff }}(\mathrm{K}), \log (\mathrm{g})(95 \%)\end{array}$ & $\begin{array}{c}\text { Spectroscopy } \\
\mathrm{T}_{\text {eff }}(\mathrm{K}), \log (\mathrm{g})(95 \%)\end{array}$ & \\
\hline $\begin{array}{l}\text { Limiting Cases } \\
\text { AMES-COND } \\
\text { AMES-DUSTY }\end{array}$ & $\begin{array}{l}- \\
- \\
1800-1900,3.5-5 \\
1900,4\end{array}$ & $\begin{array}{c}2600-2800,3.5-4 \\
2800,4.5 \\
2500-2800,3.5-4 \\
2700-2800,4.5 \\
2700,5\end{array}$ & \\
\hline $\begin{array}{l}\text { Cloud Models } \\
\text { BT-Settl } \\
\text { Burrows/A4 }\end{array}$ & $\begin{array}{l}1800,3.5 \\
1800-2000,3.6-4.25 \\
1900-2000,3.4\end{array}$ & $\begin{array}{c}1700-1900,4-5 \\
2400,3.5-4 \\
1950-2100,3.4-3.8\end{array}$ & $\begin{array}{l}- \\
-\end{array}$ \\
\hline $\begin{array}{l}\text { Models Fitting Photometry and Spectra } \\
\text { Burrows/A4 }\end{array}$ & $1950-2000,3.4-3.8$ & & \\
\hline
\end{tabular}
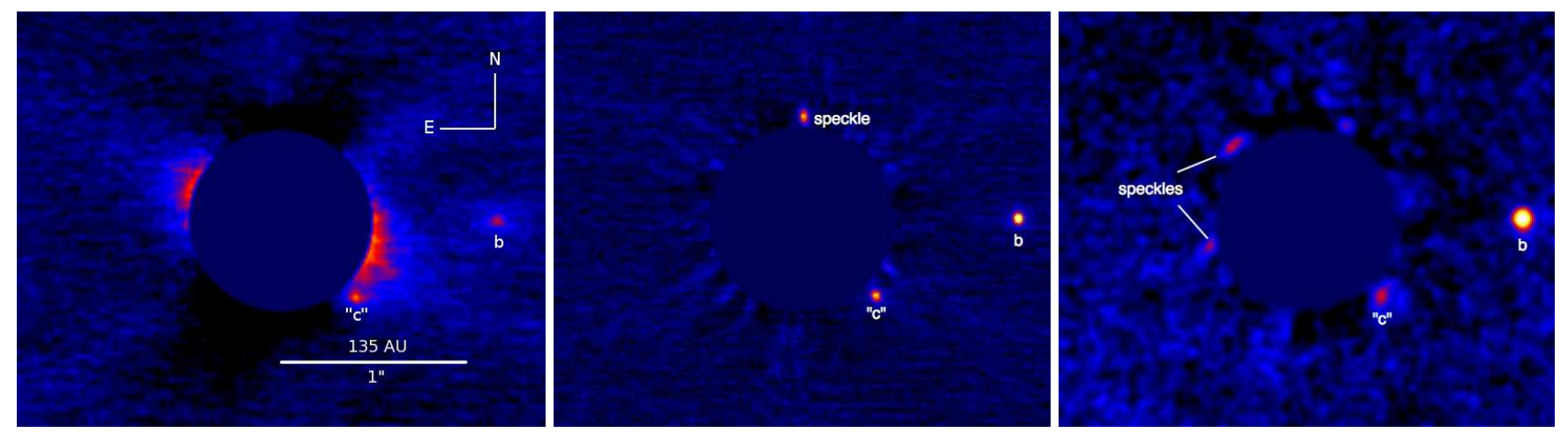

FIG. 1. - Images of ROXs 42B from $J$ (left), $K_{s}$ (middle), and $L^{\prime}$ (right) 2011 NIRC2 data. ROXs $42 \mathrm{Bb}$ and the background object "ROXs $42 \mathrm{Bc}$ " are detected in each image.
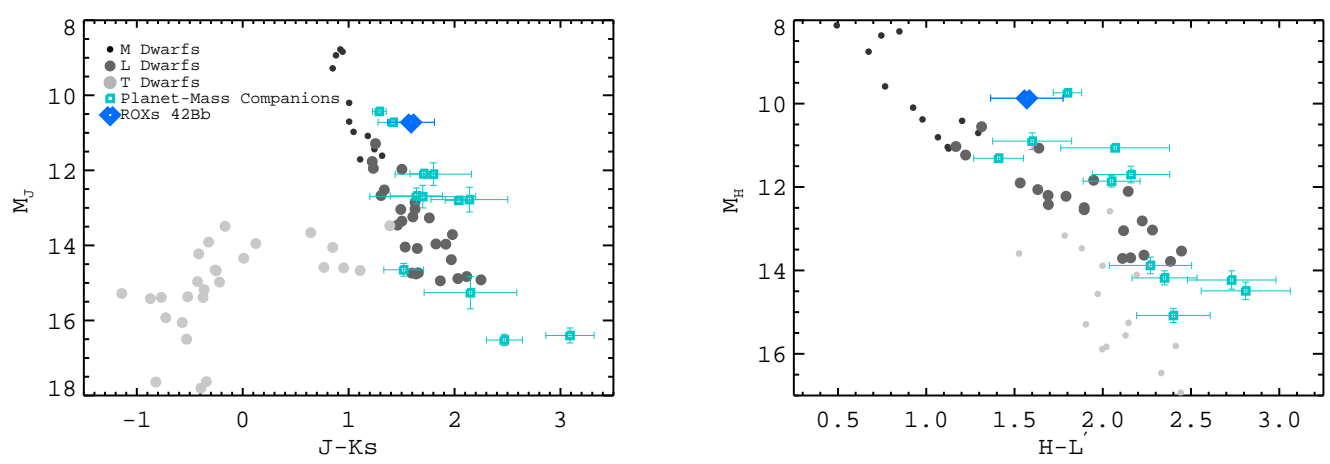

FIG. 2.- (Left) $J / J-K_{s}$ and $H / H-L^{\prime}$ color-magnitude diagrams comparing ROXs $42 \mathrm{Bb}$ 's photometry (blue diamonds) to MLT dwarfs from Leggett et al. (2010) and other planetary-mass companions (aqua squares).

mosphere models do appear to reproduce key features of ROXs $42 \mathrm{Bb}$ at these wavelengths: the depth of the $K I$ lines at $J$, the sharp drop at $1.3 \mu \mathrm{m}$, and the triangular shape of the $H$ band spectrum. 


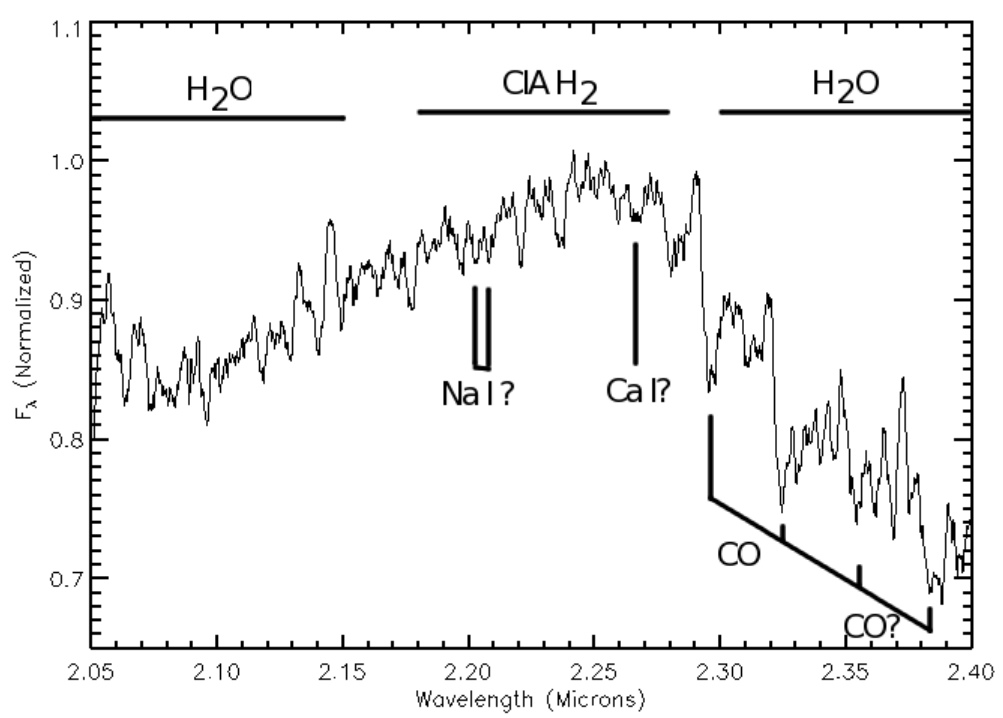

FIG. 3. - Smoothed, binned ROXs $42 \mathrm{Bb}$ continuum with detected broad molecular features $-\mathrm{H}_{2} \mathrm{O}$ and the gravity sensitive collisionallyinduced $\mathrm{H}_{2}$ feature - and the $\mathrm{CO}$ bandhead along with the locations of $\mathrm{Na}$ I and $\mathrm{Ca}$ I lines that serve as secondary gravity indicators where we do not have strong detections.
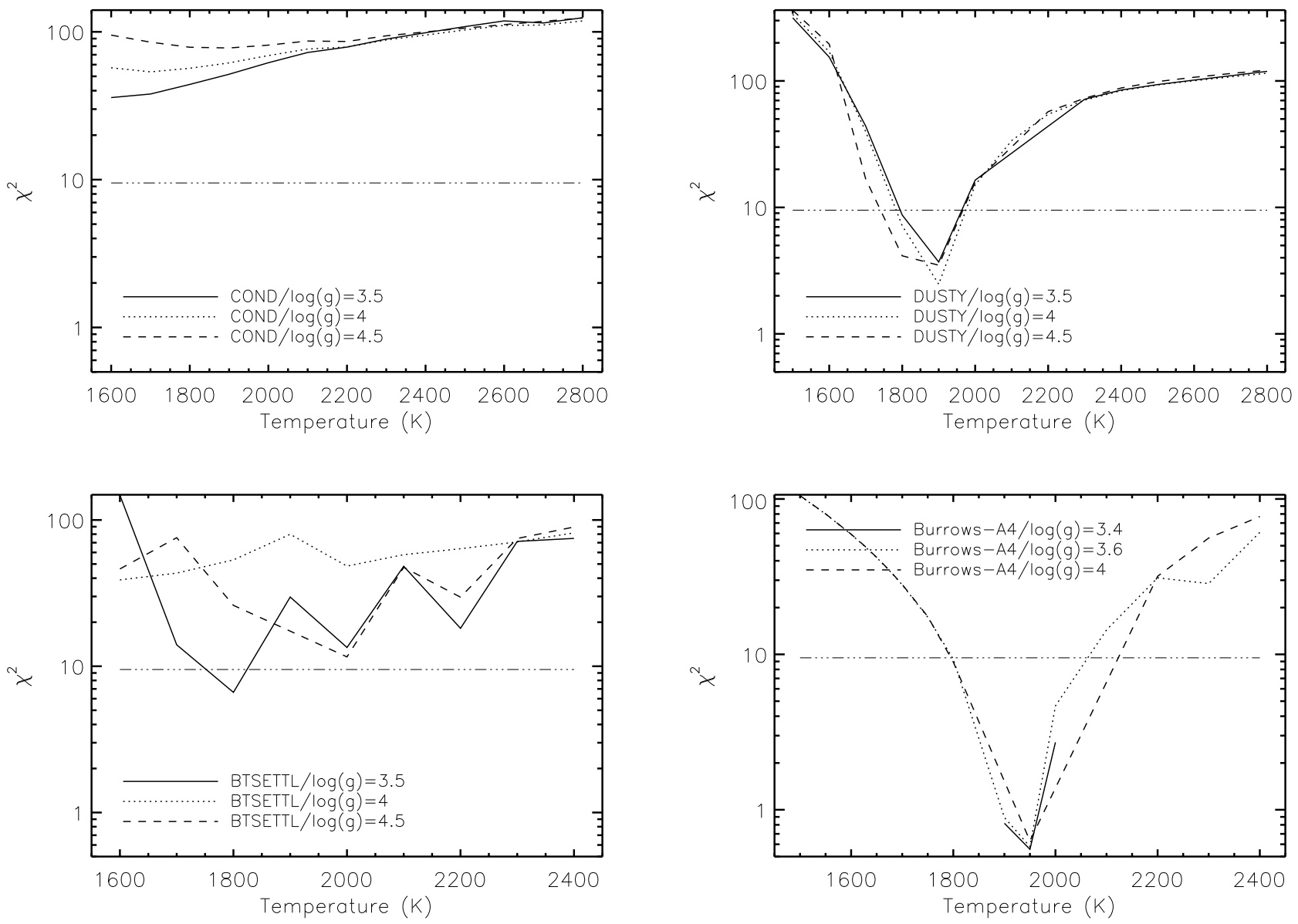

FIG. 4.- The $\chi^{2}$ distributions for fitting ROXs $42 \mathrm{Bb}$ photometry with the COND (top-left), DUSTY (top-right), BT - Settl (bottomleft), and Burrows (bottom-right) atmosphere models. The horizontal dash-three dotted line identifes the $\chi^{2}$ limit below which models agree with the data to within the $95 \%$ confidence limit. For clarity, we do not display all models of different surface gravity for the Burrows grid as the general trends match those for models with different surface gravities. 

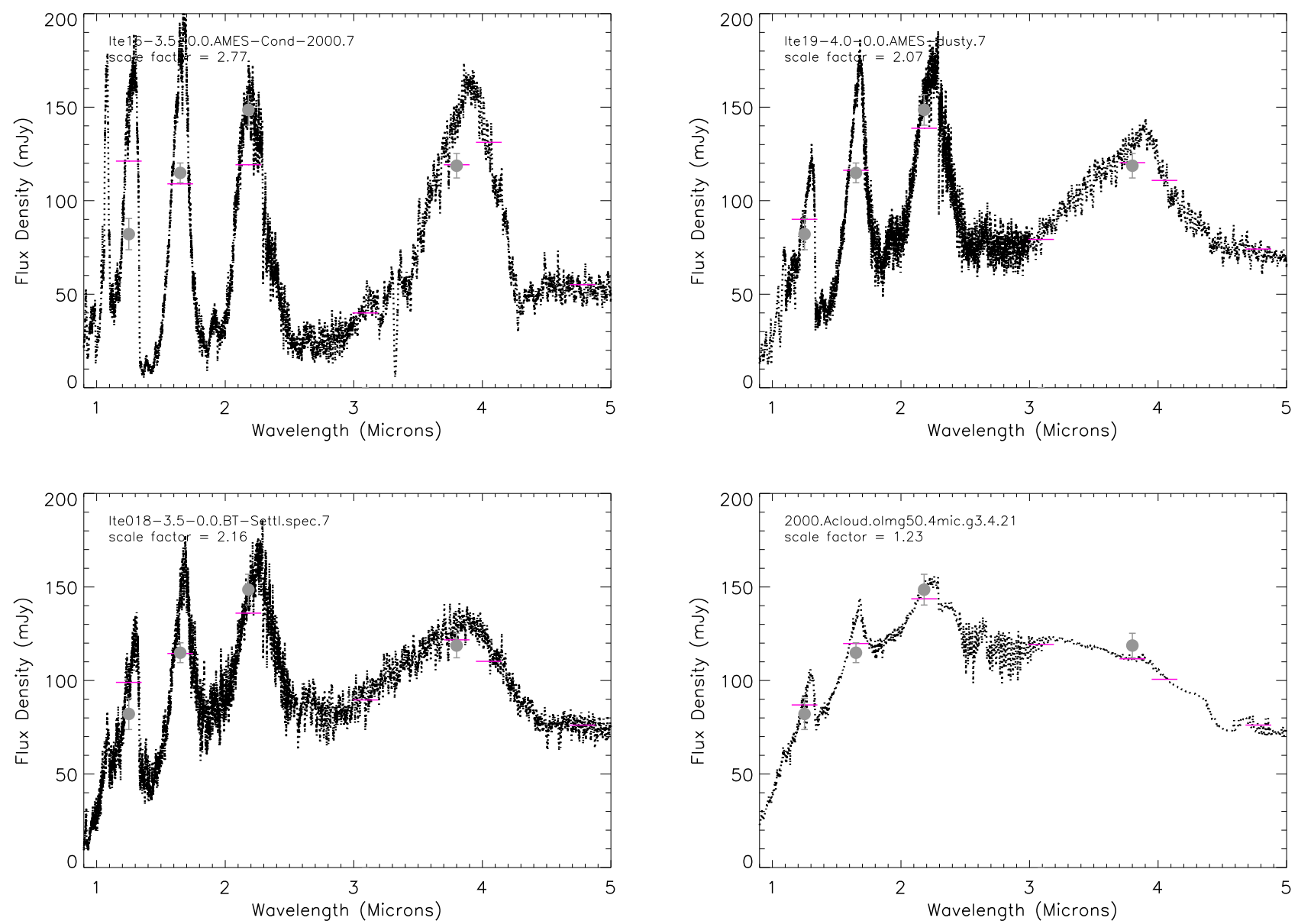

Fig. 5. - Best-fit models fits to the photometry (grey dots) for each of the four atmospheric model grids (dark lines). The horizontal magenta lines show the predicted photometry from the models at the wavelengths with ROXs 42Bb detections plus longer wavelength [4.05] and $M^{\prime}$ filters studied in Currie et al. (2013). 

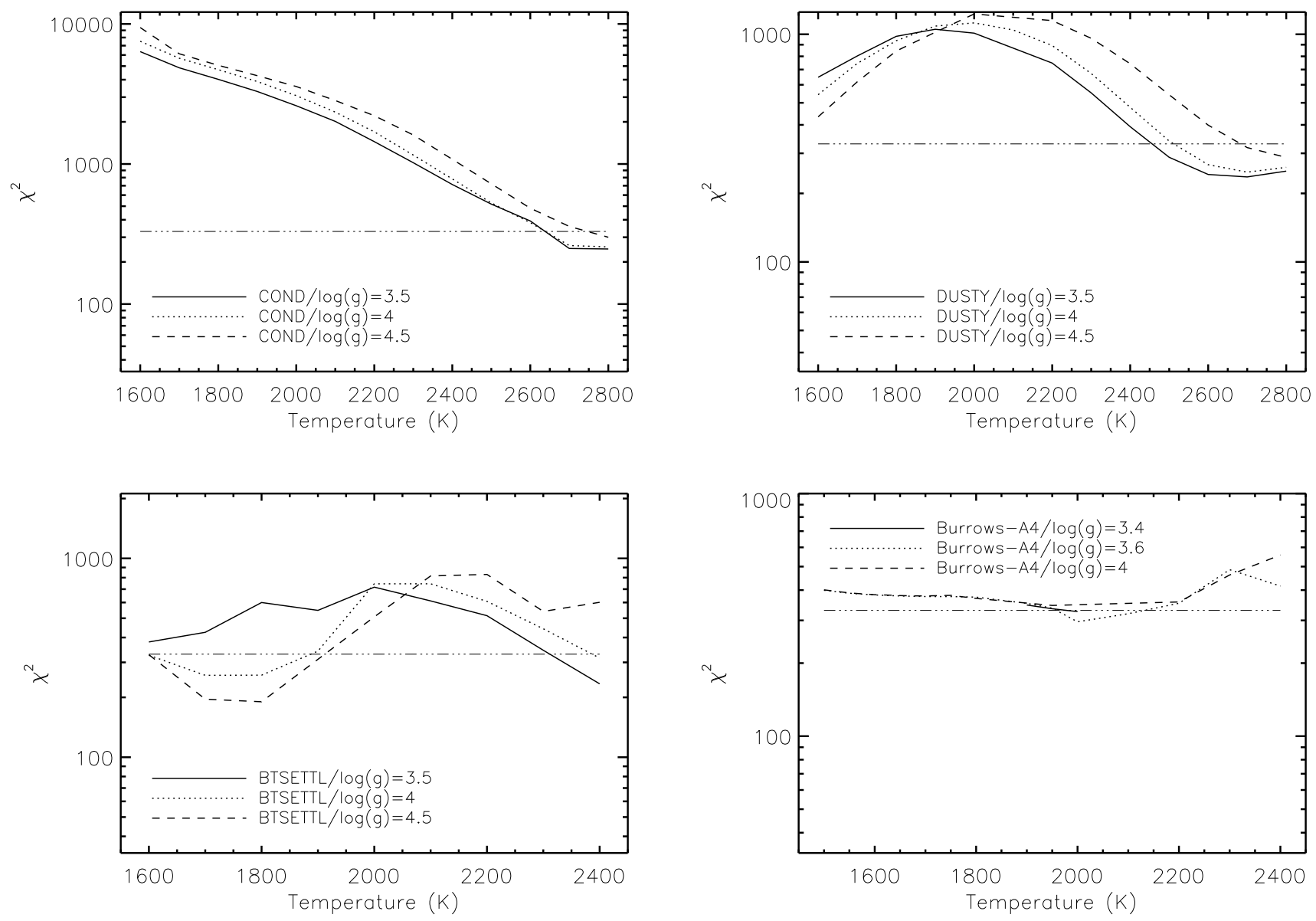

FIG. 6.- Same as Figure 4 except for the $K$-band spectrum. 

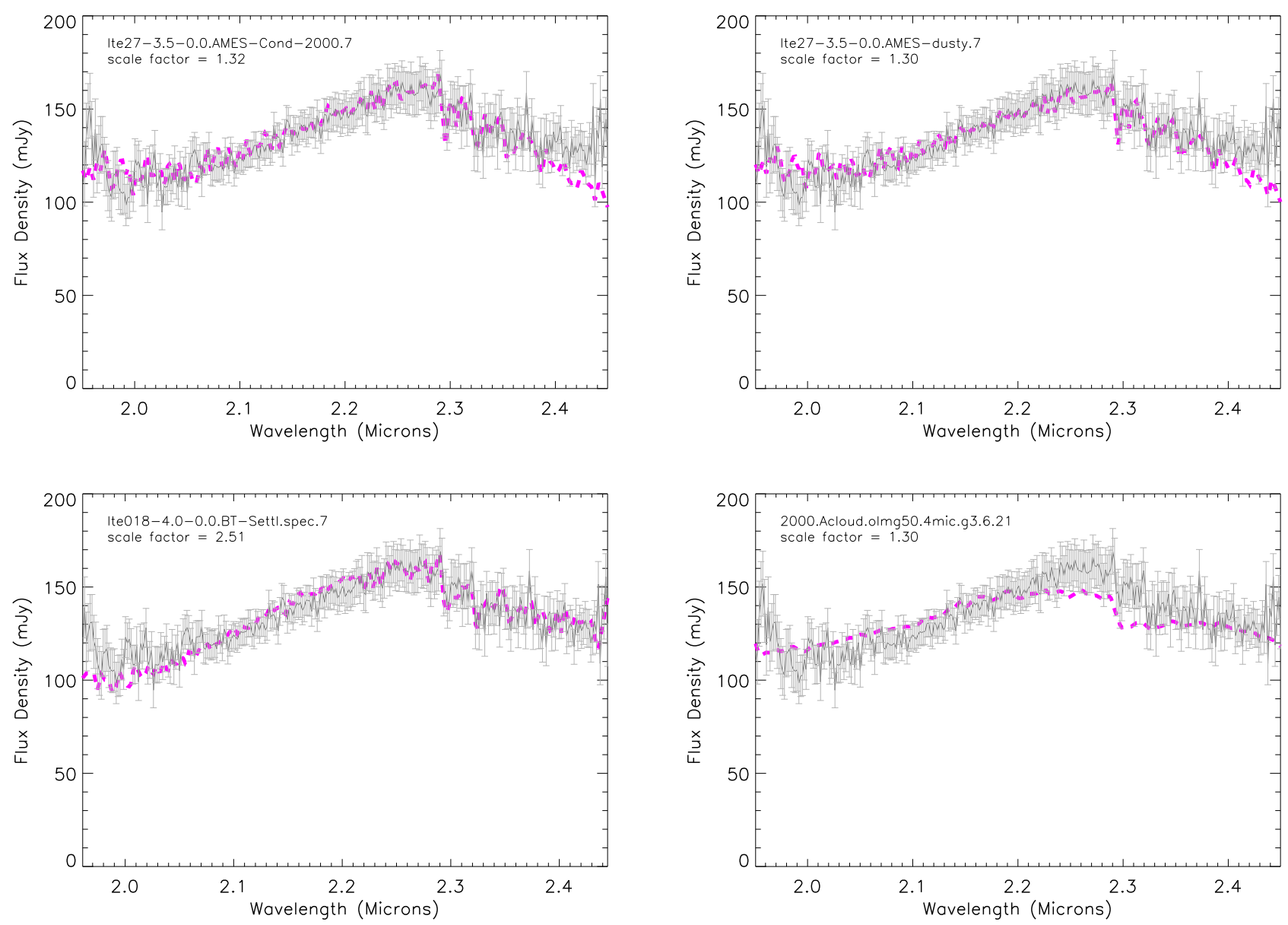

FIG. 7.- Same as Figure 5 except for the $K$-band spectrum. 

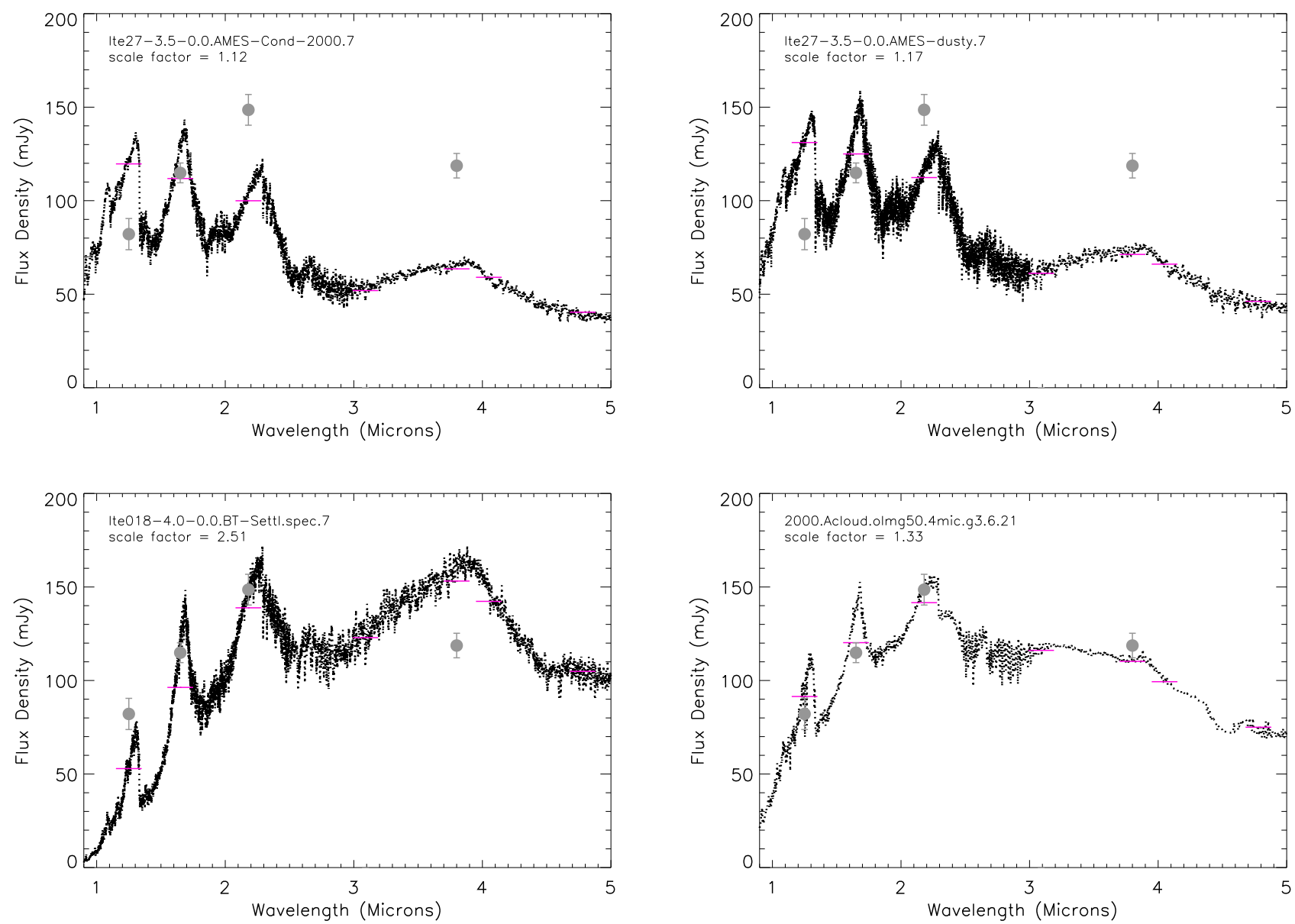

FIG. 8. - Comparisons between models that best fit the ROXs $42 \mathrm{Bb} K$-band spectrum and the ROXs $42 \mathrm{Bb}$ photometric data. All $C O N D$ models, including those that accurately reproduce the $K$-band spectrum, fail to reproduce the companion's photometric data. The DUSTY and BT-Settl models that best fits the spectrum fail to match the companion's photometry. 

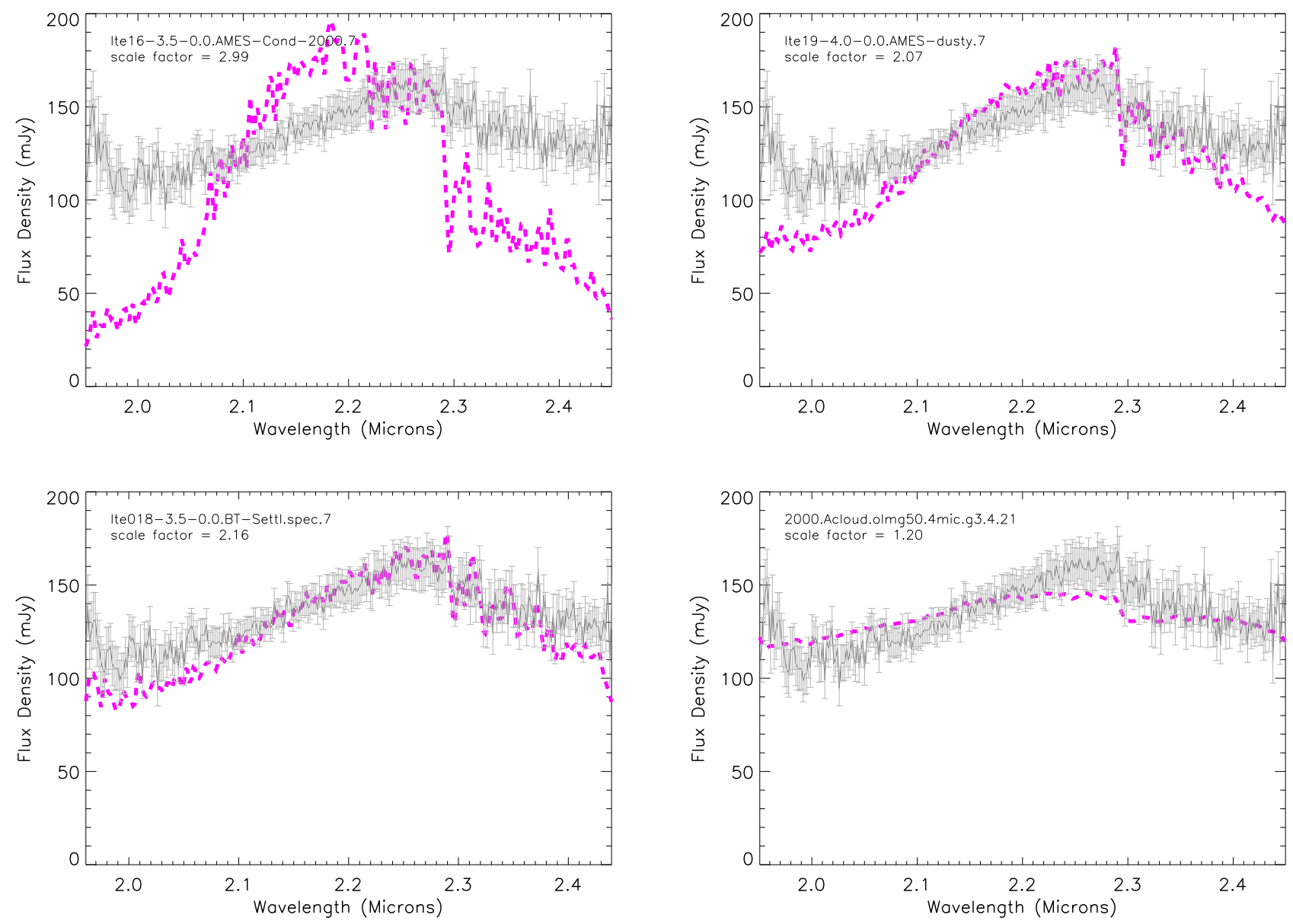

FIG. 9.- Same as Figure 8 except comparing models that best fit the ROXs $42 \mathrm{Bb}$ photometry to ROXs $42 \mathrm{Bb}$ 's $K$-band spectrum. The $C O N D$ and DUSTY models clearly mismatch the $K$-band spectral shape. The BT-Settl model predicts slightly too narrow of a $2.2 \mu m$ peak, while the Burrows model fares better. A small range of parameter space $-T_{\text {eff }}=1950-2000 \mathrm{~K}, \log (\mathrm{g})=3.4-3.8-$ matches both the photometry and spectrum. 